\title{
A Cooperative ARQ for Delay-Tolerant Vehicular Networks
}

\author{
Julián Morillo-Pozo, Oscar Trullols, Jose M. Barceló, Jorge García-Vidal \\ Department of Computer Architecture (DAC), UPC, Barcelona, Spain \\ \{jmorillo,trullols,joseb,jorge\}@ac.upc.edu
}

\begin{abstract}
$^{1}$
This paper proposes a Cooperative ARQ protocol to be used in delay-tolerant vehicular networks. The proposed scheme has been implemented and evaluated through an experimental testbed, showing that packet losses in transmissions from an access point to cars can be reduced to the half without any cost as long as cooperation takes place on areas where connectivity with access points is not present. In these areas, cars in a platoon recover from other cars packets that they have failed to receive from the access point.
\end{abstract}

\section{Introduction}

Vehicular Ad-hoc NETworks (VANETs) are a particular case of MANETs in which nodes are vehicles that move following specific patterns (i.e. roads). Important applications of VANETS are: Transportation-related applications and Convenience and Personalized applications including Internet access, hot-spots access, gaming, sharing files or $\mathrm{P} 2 \mathrm{P}$ services.

In this paper we focus on delay-tolerant applications, in which cars download information from Access Points (APs) placed on the road.

VANETs are networks characterized by intermittent connectivity and rapid changes in their topology. In the scenario considered, vehicles accessing an AP have few seconds to download information in an environment with high losses. Measurements of UDP and TCP transmissions of vehicles in a highway passing in front of an AP moving at different speeds report losses on the order of $50-60 \%$ depending on the nominal sending rate and vehicle speed; see [1].

In this harsh environment, innovative communication techniques are needed. We believe that cooperative techniques can be beneficial in order to improve the performance of this type of networks and

${ }^{1}$ This work has been supported by Spanish Ministry of Science and Technology under grant TSI2007-66869-C02-01 and by the NoE EuroFGI of the VI FP of the UE under VNET-3 project. applications. The main objective of this paper is to test this hypothesis.

The main contributions of our work are the following:

(i) We propose a variation of the Cooperative ARQ (C-ARQ) scheme to be used in vehicular networks where cars download delay-tolerant information from APs on the road, suffering an intermittent connectivity. Cooperation among cars is established in the dark areas, where connectivity with the APs is lost.

(ii) To evaluate the proposal, we have built an experimental prototype based on IEEE 802.11 technology, which runs in a real urban environment.

The main outcome of our research is that the proposed protocol can effectively reduce the packet losses of transmissions from access points to cars in a platoon. We have demonstrated, with a prototype that uses a very simple implementation of the mechanism, that an almost optimal performance can be achieved in the sense that, given the packet receptions on each car in the platoon, each car is able to recover all the packets it has lost from the access point from the other cars provided that they have them. The experimental results give promising improvements to take into account for future research on the field.

\section{Related work}

A performance study in term of losses when vehicles enter the coverage of an access point in a highway and exchange UDP and TCP packets is presented in [1].

Most of the work related to opportunistic vehicular networks deal with opportunistic forwarding strategies, in which nodes schedule the forwarding of packets according to opportunities; see [2], [3], [4]. This scheduling may be based on: historical path likelihoods, [2], packet replication, [3], or on the expected packet forwarding delay, [4]. These proposals take as a point of reference epidemic routing [6]. Their main objective is to optimize contact opportunities to 
forward packets in intermittent scenarios, but they do not consider how to optimize the transference of such information given that you have contacted another node. Our work follows the Infostation model, in which nodes transport data, and deliver their information during contact times; see [5]. In our case hot-spots distributed along roads act as Infostations, while gaps between Infostations are used to interchange packets with other nodes.

Cooperative ARQs are schemes which increase link reliability in data link protocols through the use of node cooperation; see [9], [10], [11], [12]. In [10] authors describe a scheme for improving loss resilience with diversity, focusing on wireless local area networks (WLANs). In [9], authors propose a twophase communication using a relay node. In [11] authors present a generalization of Hybrid-ARQ where retransmitted packets do not need to come from the original source radio but could instead be sent by relays that overhear the transmission. The job reported in [12] presents a novel frame exchange mechanism between a node and its cooperators for $\mathrm{C}-\mathrm{ARQ} / \mathrm{FC}$ (Cooperative ARQ with Frame Combining).

\section{A Cooperative ARQ for Delay-Tolerant Vehicular Networks}

In this section we introduce a novel Cooperative ARQ scheme that allows nodes of an 802.11-based delay-tolerant vehicular network to work cooperatively in order to increase the delivery rate of all of them in packets received from a fixed AP.

Consider Figure 1 in which vehicles want to download information from the Internet through APs distributed along a road. Due to the harsh conditions produced in VANETs, the losses produced in such environment are high. Reference [1] reports experiments on a highway in which vehicles passing in front of an AP moving at different speeds have losses on the order of $50-60 \%$ depending on the nominal sending rate and vehicle speed.

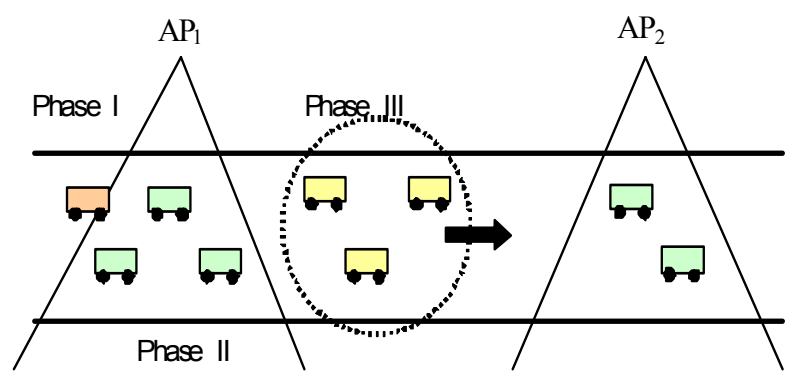

Figure 1. Network Scenario
We propose a scheme to decrease this high level of packet losses which uses the so-called Cooperative ARQ protocol. The scheme operates into three phases, see Figure 1:

\subsection{Association phase}

Suitable mechanisms for detecting in-range AP, association and authentication of vehicles reaching a given AP must be provided. Those mechanisms can have a major importance on the overall performance, but are not specific of the use or not of cooperation, and thus we leave them out of the scope of this paper. It can be assumed, for example, that vehicles are equipped with WAVE (Wireless Access in Vehicular Environments) IEEE $802.11 \mathrm{p}$ cards. WAVE architecture provides mechanisms to access WAVE Base Stations (APs) in vehicular networks.

On the current implementation of the prototype used for the testbed we use 802.11 technology, we have not considered security issues and the association with the AP has been made very simple: the AP is continually transmitting numbered packets addressed to each car in the experiment and a vehicular node is considered associated with the AP in the moment it receives a packet from the AP (it enters into the coverage area).

\subsection{Reception phase}

Nodes are operating in this phase while they are on the coverage area of the AP. During this phase, nodes will request the information blocks to be downloaded and the AP will transmit them to the vehicular nodes.

In our prototype, the exact request mechanism is not implemented, and this phase starts with the reception of the first packet from the AP and finishes when no packets have been received for a given time period.

While in this phase, vehicular nodes receive data from the AP. Each car receives its data but also buffers the packets addressed to other cars in the platoon that consider it as cooperator. The cooperation relationship is established through the exchange of HELLO messages broadcasted periodically by the vehicular nodes. The first function of a HELLO message sent by a node $x$ is to allow other nodes to know about the presence of $x$. Other vehicular node $y$ in the platoon will add $x$ to its list of cooperators (if $x$ is not already a cooperator of $y$ ) when receiving this HELLO message. The second function of a HELLO message sent by a node $x$ is to notify other nodes about the fact that they have to act as cooperators of $x$. For this second function, each HELLO message contains the list of cooperators of the sending node. In our example, the 
next HELLO message sent by $y$ will contain $x$ in the list of cooperators. In this way, $x$ will be aware of the fact that $y$ considers it as cooperator and will act accordingly (buffering packets addressed to $y$ for a later possible cooperation). The list of cooperators contained in the HELLO messages also indicates the order in which cooperators should act in the Cooperative-ARQ phase: this is to avoid collisions; when a node requests a packet to its cooperators, each cooperator will wait a fixed back-off depending on this assigned order, before sending the packet. Note that we do not focus on the cooperators selection algorithm, so this is left out of the scope of this document. In our work, only one hop neighboring nodes can be selected as cooperators, although other schemes can be envisioned.

In the considered scenario, data flow is always from the AP to the vehicular nodes, and no retransmissions are used. We avoid retransmissions at the hope that other cars in the platoon (i.e. cooperators) will receive packets incorrectly received by the destination and will help it in the Cooperative-ARQ phase, without the need of wasting the useful time in coverage with the AP in retransmissions. In this way the channel can be used by the AP to transmit as much new data addressed to the cars as possible, thus reducing the downloading time and increasing the effective data rate. Of course, a retransmission scheme (possibly adaptive with respect to the number of cooperators) would be needed in a real system, but the study of that is left for future work.

\subsection{Cooperative-ARQ phase}

When the cars leave the AP range, they enter into the Cooperative-ARQ phase. In our prototype this phase starts when the timeout from the last received packet from the AP expires (5 seconds in the current implementation). At this point, every node checks which packets it has failed to receive correctly from the AP and starts to request them to other vehicular nodes (i.e. to its cooperators), in an attempt to recover all packets from the first to the last received from the AP. The process is the following: (i) A node $x$ broadcasts a REQUEST packet for each packet that is has failed to receive from the AP. (ii) When receiving this REQUEST, each cooperator of $x$ will check if it has the requested packet buffered (it has received the packet correctly from the AP in the previous phase). (iii) If it has the packet, it will wait a fixed time depending on the order of cooperation assigned by $x$ through the HELLO messages as explained on previous subsection, and will send the packet to $x$ (unless other cooperator sends it before).

This process will be repeated for each missing packet. When the final of the list of missing packets is reached, the vehicular node will start again from the beginning of the actualized (shorter) list of missing packets. A node stops to issue requests of missing packets when it has recovered all of them or when it enters in range of a new AP, meaning that it comes into reception mode (Reception phase of the protocol operation), and the whole cycle starts again.

Note that the operation in this phase can be optimized in many aspects. For example, one optimization that arises directly is to include in the REQUEST messages all the missing packets, instead of sending a REQUEST for each one. In this way, although it could have some similarities, the cooperation would not behave as epidemic routing (see [6]) in which nodes carry and forward packets for other nodes. Cooperative-ARQ objective is to improve performance given that the neighborhood of a node has received packets directed to that node.

\section{Experimental setting}

A performance evaluation of the proposed mechanism has been performed using a real implementation. The tests were performed in the urban scenario depicted in Figure 2. The AP was located in the position marked as AP in Figure 2 and consisted in a desktop PC equipped with a Proxim external PCI wireless antenna located in an office in the first floor of the building. The antenna was located on the window of this office.

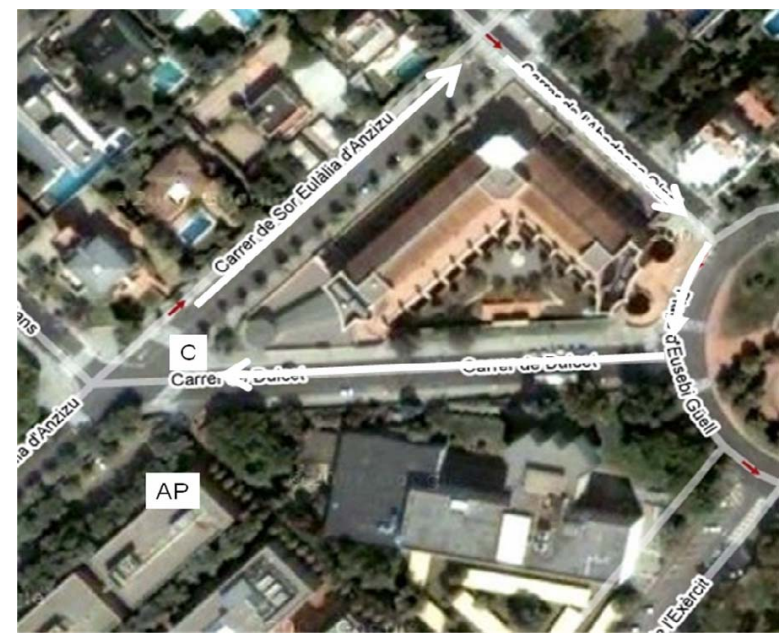

Figure 2. Map of the testbed

On the other hand, three mobile vehicular nodes were used, consisting of three laptops (Toshiba Satellite Pro A120 model) equipped with Cisco Aironet AIR-CB21AG-E-K9 802.11a/b/g PCMCIA wireless adapters, each of them transported by a car. The three cars followed the path marked with the white 
arrows in Figure 2 all together at an average speed of about $20 \mathrm{Km} / \mathrm{h}$. for a total number of 30 rounds. We have named them as Car 1 (the first), Car 2 (the car in the middle) and Car 3 (the last).

The implementation of the mechanism was done using Click Modular Router [7] and all the cards were controlled by a MadWiFi [8] driver in monitor mode and with retransmissions disabled. All transmissions (i.e. AP to Car, Car to Car) were performed using $802.11 \mathrm{~g}$ at $1 \mathrm{Mbps}$.

The AP transmitted three different data flows addressed to each car on the experiment consisting of 5 ICMP Echo Request messages per second with an ICMP payload of 1000 bytes, each one. During the experiments we captured all the received traffic on each laptop for its analysis and post-processing to obtain the results exposed in next section.

We have performed the tests on an urban scenario for its easiness of deployment in contrast to a highway scenario. Of course, losses will be lesser than the reported in [1] because of the speed of vehicular nodes and the lower data rate we employ for the tests. However, this simple scenario allows us to show how cooperative techniques and more precisely, Cooperative ARQ can help on the improvement of these kinds of networks.

\section{Experimental results}

Firstly, we present in Table 1 the average values on packet losses obtained along the 30 rounds performed on the experiment. Together with the mean absolute values, we show the percentages of losses without and with the cooperative ARQ mechanism.

Table 1. Average values on the number of packets received and lost in the three cars.

\begin{tabular}{|c|l|c|c|c|}
\hline \multicolumn{2}{|l|}{} & \multicolumn{1}{|l|}{$\begin{array}{l}\text { Tx by the } \\
\text { AP }\end{array}$} & $\begin{array}{l}\text { Lost before } \\
\text { coop. }\end{array}$ & $\begin{array}{l}\text { Lost after } \\
\text { coop. }\end{array}$ \\
\hline \multirow{3}{*}{1} & Mean & 130.4 & $\begin{array}{c}30.5 \\
(23.4 \%)\end{array}$ & $\begin{array}{c}13.7 \\
(10.5 \%)\end{array}$ \\
\cline { 2 - 5 } & Std. Dev. & 17.7 & 12.9 & 9.1 \\
\hline \multirow{4}{*}{2} & Mean & 143.0 & 38.4 & 24.8 \\
& & & $(26.9 \%)$ & $(17.3 \%)$ \\
\cline { 2 - 5 } & Std. Dev. & 18.6 & 12.4 & 11.8 \\
\hline \multirow{3}{*}{3} & Mean & 121.4 & 34.7 & 19.1 \\
& & & $(28.6 \%)$ & $(15.7 \%)$ \\
\cline { 2 - 5 } & Std. Dev. & 17.2 & 15.5 & 14.4 \\
\hline
\end{tabular}

As can be seen in Table 1, all three cars present an improvement on the reliability on the link between the AP and themselves. Especially striking is the case of car 1 , where a reduction of more than $50 \%$ in the number of lost packets is achieved. It seems strange that the poorer results are obtained for car 2. This fact, however, can be explained, as we will see, by the environment and by the different behavior of the three cars along the experiment (distances between them, etc.). In other tests, not shown here, car 2 normally achieved the best performance. This is a normal result as car 2 is the car located in the middle of the platoon and can benefit from the cooperation coming from car 1 on the first range of packets it should have received from the access point while entering the coverage area and from the cooperation of car 3 on the last packets that it should have received while leaving the coverage area.

Now that we have seen the mean values obtained on the experiment, let us focus into the details to explain them and study the probabilities of reception of packets on the different cars. In Figure 3 the probability of reception for the three different cars of packets addressed to car 1 is shown. Three different packet reception regions can be defined: Region I corresponds to car 1 being at the beginning of the AP coverage area while cars 2 and 3 are just entering it. Region II corresponds to the car 2 and/or car 3 on the coverage area together with car 1 . In Region III, car 1 is leaving the coverage area while car 2 and/or 3 are still there.

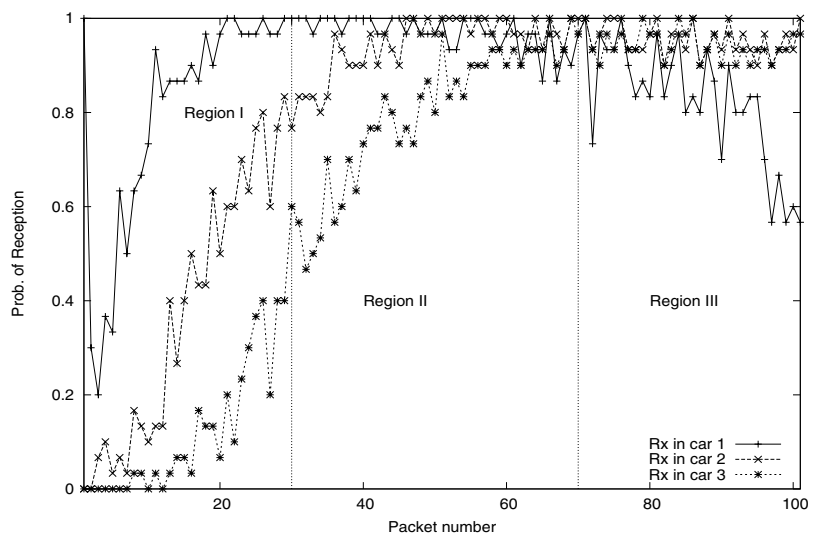

Figure 3. Probability of reception in packets addressed to car 1.

Figure 3 depicts how, when car 1 is entering the coverage area (Region I), its probability of reception is much better of that in car 2 and even better compared with that in car 3 , which implies that car 1 will receive little cooperation from 2 and 3 for this range of packets. When car 1 starts to leave the coverage area (Region III), however, we can see how both car 2 and car 3 have higher reception probability, suggesting that car 1 will benefit from cooperation for this range of packets. It is also important to note that, while on Region I of the figure, car 2 and car 3 perform quite different, on Region III their probabilities of reception are almost the same. We argue that this is because of the behavior of the different drivers. The fact is that the 
driver in car 2 was the least experienced, thus meaning that at corner marked as $\mathrm{C}$ on Figure 2, car 3 became very close to car 2 in almost all rounds, making their reception conditions on the street before the corner (after turning to the right on corner C) quite similar.

Figure 4 presents the same results but for car 2 . We can observe on Region I of Figure 3 (first packets) that, as expected, car 1 has better reception conditions, so car 2 will benefit from cooperation coming from car 1 . For the last packets (Region III of the figure) a better reception probability on car 3 was expected. However, due to the pattern on the cars' movement explained before, this is not the case and the reception condition for car 2 and 3 are almost the same.

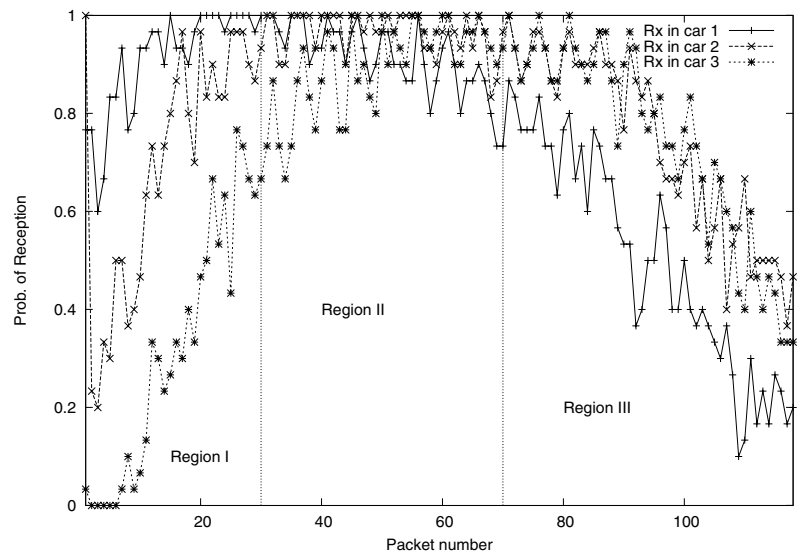

Figure 4. Probability of reception in packets addressed to car 2.

On Figure 5 we can observe how, when car 3 is entering into the coverage area (Region I), both cars 1 and 2 experience better packet reception probabilities, making them good cooperators. When car 3 is leaving the coverage area (Region III), however, car 1 has very worst reception conditions as it is almost out of the coverage area.

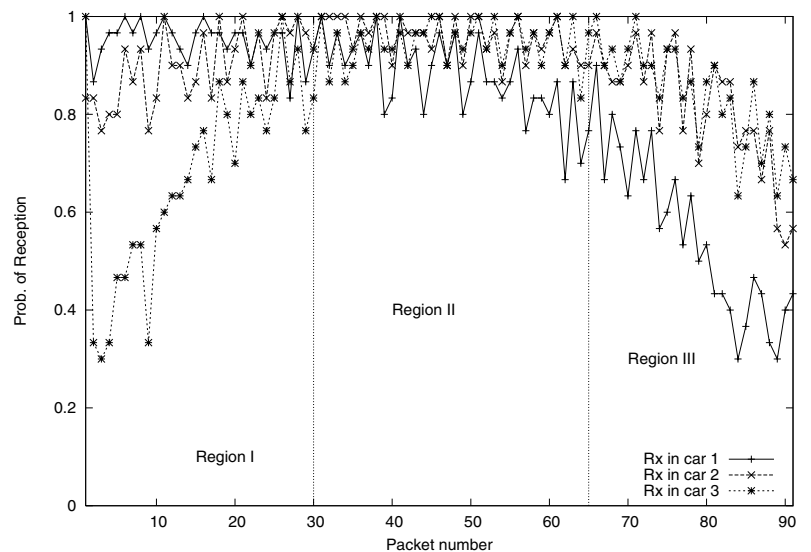

Figure 5. Probability of reception in packets addressed to car 3.
The next figures (Figures 6, 7 and 8) present interesting results taking a different approach. In them the probability of correct reception using C-ARQ (i.e. after cooperation) is compared with the joint probability of reception of the different packets in car 1,2 or 3 in order to establish if the C-ARQ mechanism implemented and tested works properly and the effectiveness of it. In all the figures, the two curves are almost coincident indicating that the protocol works almost optimally in the sense that the destination car is able to recover all the packets that have been received in any of the cars in the platoon. Let us focus now on Figure 6 and analyze it in conjunction with Figure 3. As can be seen, the curve in Figure 6 for Region I has the same shape of the Rx in car 1 curve in Region I in Figure 3. This is because in this case all received packets were received directly by car 1 . In Region II, car 1 experiences very good reception conditions, so it will not need cooperation for this range of packets. For packets between 60 and 100 (Region III), however, it can be seen on Figure 3 how the probability of reception in car 1 decreases greatly (it is leaving the coverage area). But, thanks to $\mathrm{C}-\mathrm{ARQ}$, it is able to recover most of the packets helped by car 2 and 3: note that the shape of Region III of Figure 6 is almost coincident with the Rx in car 2 and $\mathrm{Rx}$ in car 3 curves in Region III of Figure 3. Here is the key idea behind the mechanism: it exploits the diversity that can be achieved thanks to the different cars on a platoon and performs as well as a virtual car which uses the better reception conditions of all of them.

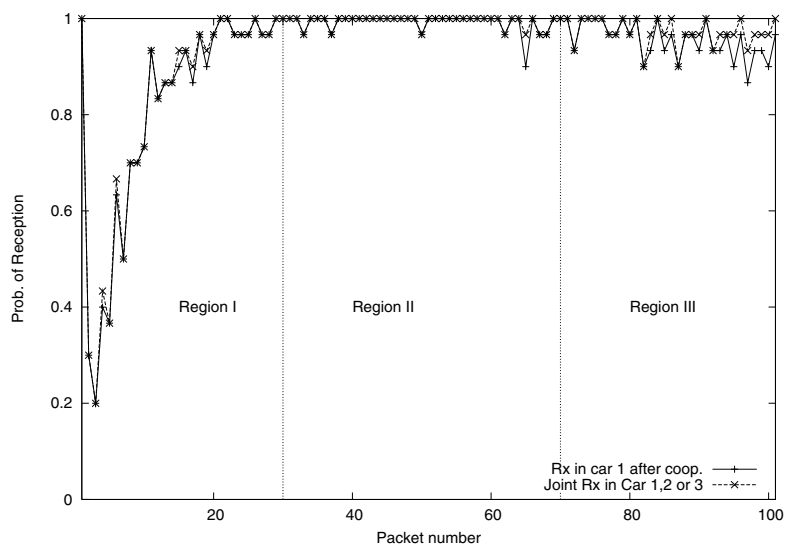

Figure 6. Probability of reception with C-ARQ in car 1.

A similar analysis can be done for Figure 7 and using Figure 4 for comparison purposes. We can see how the reception in car 2 after the cooperation phase in Figure 7 for Region I performs more or less like car 1 in Figure 4, which means that car 2 has benefit greatly from cooperation coming from car 1 . 


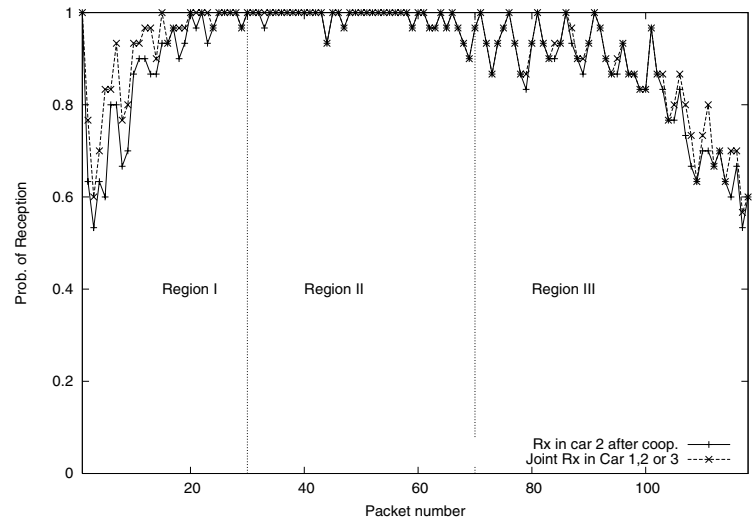

Figure 7. Probability of reception with $\mathrm{C}-\mathrm{ARQ}$ in car 2.

On Figure 8 it can be seen how car 3 benefits from cooperation for the first 30 packets range. For the last packets, little cooperation can be used, as long as car 3 is the last car in leaving the coverage area.

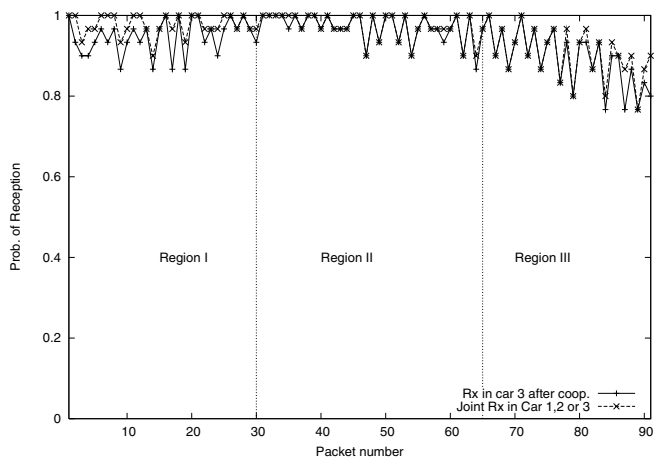

Figure 8. Probability of reception with $\mathrm{C}-\mathrm{ARQ}$ in car 3.

\section{Conclusions and further work}

This paper has presented a novel mechanism to be used in delay-tolerant vehicular networks based on a Cooperative ARQ protocol. An important issue on these kinds of networks is that vehicles accessing an AP have few seconds to download in a harsh environment with high level of losses. The main objective of the proposed scheme is to reduce these packet losses.

We have studied through a real implementation and a testbed in an urban scenario how the proposed mechanism can effectively reduce the packet losses.

Many questions are left for future work and remain as open issues. For example, an algorithm for selecting the optimal cooperators has not been addressed. Retransmissions schemes for these kinds of systems need also to be studied. Moreover, we have indicated how the way in which vehicular nodes request the lost packets to their cooperators can be optimized but the behavior of this approach needs to be studied. Even more important is to study how the presented loss reduction can reduce the number of APs that a vehicular node needs to visit to download a file or how it can allow to increment the bit rate used by the APs.

\section{Acknowledgements}

We would like to thanks Professor Ian Akyildiz for his valuable comments and discussions on this work.

\section{References}

[1] J. Ott, D. Kutscher, "Drive-thru Internet: IEEE 802.11b for 'Automobile' users”, IEEE INFOCOM 2004.

[2] J. Burguess, B. Gallagher, D. Jensen and B.N. Levine, "MaxProp: Routing for Vehicle-based Disruption Tolerant Networks", 25th Conference on Computer Communications (IEEE INFOCOM), Barcelona, Spain, April 2006.

[3] A.Balasubramanian and B. N. Levine and A. Venkataramani, "DTN Routing as a Resource Allocation Problem" , ACM SIGCOM'07, Kyoto, Japan, August 2007.

[4] J. Zhao and G. Cao, "VADD: Vehicle-Assisted Data Delivery in Vehicular Ad Hoc Networks", 25th Conference on Computer Communications (IEEE INFOCOM), Barcelona, Spain, April 2006.

[5] T. Small and Z. J. Haas, "The Shared Wireless Infostation Model - A new Ad Hoc Networking Paradigm (or Where there is a Whale, there is a Way)", MobiHoc'03, Annapolis, Maryland, USA, June 2003.

[6] Vahdat and D. Becker, "Epidemic Routing for Partially Connected Ad Hoc Networks", Technical Report CS-200006, Duke University, April 2000.

[7] Click

Modular

Router:http://www.read.cs.ucla.edu/click/

[8] MadWifi: Multiband Atheros Driver for WiFi. http://madwifi.org/

[9] Bin Zhao, Matthew C. Valenti, "Practical Relay Networks: A Generalization of Hybrid-ARQ", IEEE Journal on Selected Areas in Communications, vol. 23, no. 1, Jan. 2005.

[10] Miu, H. Balakrishnan, C. E. Koksal, "Improving Loss Resilience with Multi-Radio Diversity in Wireless Networks", ACM Mobicom 2005, September 2005.

[11] M. Dianati, X. Ling, K. Naik, X. Shen, "A Node Cooperative ARQ Scheme for Wireless Ad-hoc Networks", IEEE Wireless Communications and Networking Conference, 2005.

[12] J. Morillo, J. Garcia-Vidal, "A Low Coordination Overhead C-ARQ Protocol with Frame Combining", $18^{\text {th }}$ Annual IEEE International Symposium on Personal, Indoor and Mobile Radio Communications (IEEE PIMRC 2007), Athens, Greece, September 2007. 